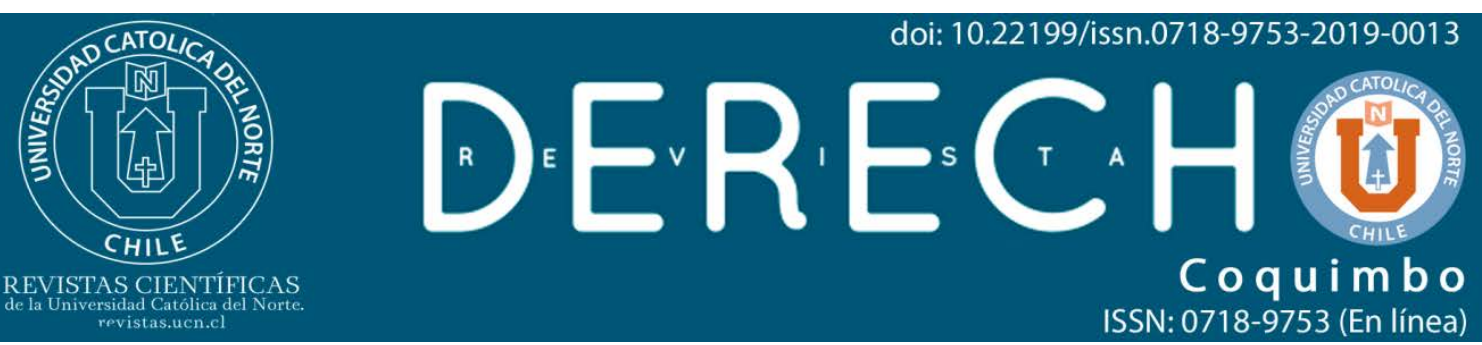

\title{
Factores limitantes del diálogo entre los procesos de ense- ñanza-aprendizaje y el contenido jurídico, en la educación del derecho
}

\section{Limiting factors of the dialogue between the teaching-learning pro- cesses and the legal content, in the education of the right}

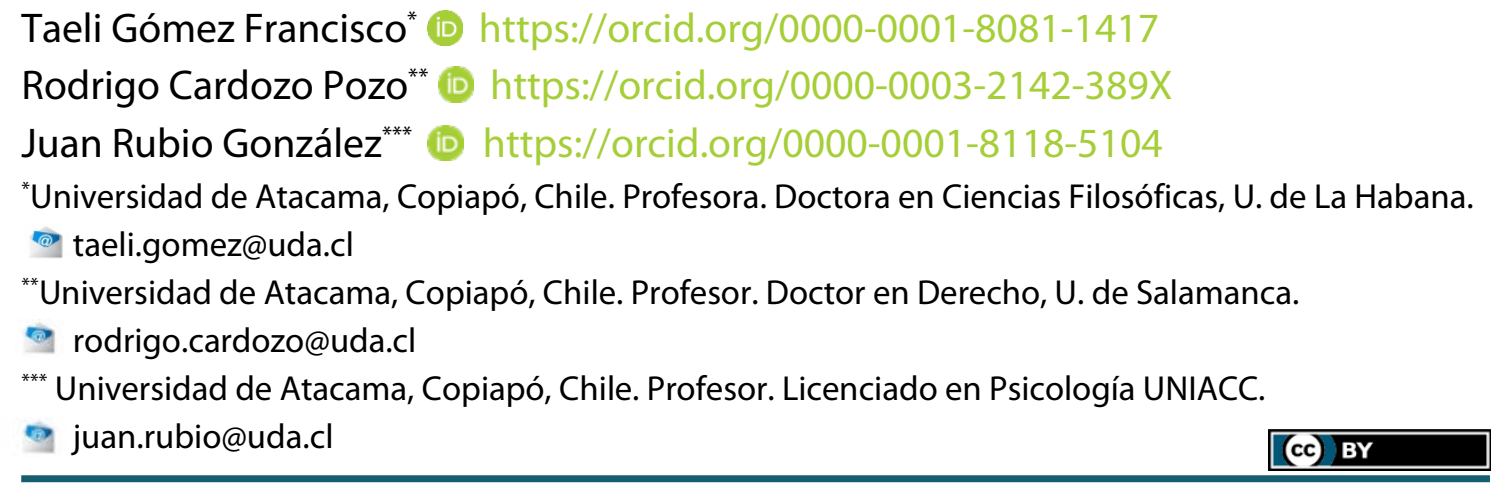

Resumen:

El propósito es demostrar la importancia que adquiere, para la enseñanza de las ciencias jurídicas, el cómo se relaciona el estudiante con el objeto de conocimiento jurídico o contenido a aprender. En él, se explican algunos de los límites que existen para que esa relación se dé de manera dialogante y activa, y se plantea por qué el estudiante no interactúa con el sistema teórico jurídico. Todo, con el objeto de superar la reproducción y asimilación pasiva de contenidos. La conclusión central demuestra las debilidades que se deben atender para superar la relación pasiva del estudiante con el objeto de conocimiento educativo jurídico, y cuáles son sus proyecciones para un aula democrática.

Palabras Clave: Enseñanza del derecho; Epistemología jurídica; Contenido jurídico.

\begin{abstract}
:
The purpose is demonstrate the importance acquired -for the teaching of the legal sciences- how the student relates to the object of legal knowledge or content to be learned. It explains some of the limits that exist for this relation as an active dialogue, and there is suggested why the student is not related to the legal theoretical system. All of this, in order to overcome the reproduction and passive assimilation of contents. The central conclusion demonstrates the weaknesses that must be addressed in order to overcome the passive relationship of the student with the object of legal educational knowledge, and what are their projections for a democratic classroom.

Keywords: Law teaching; Legal epistemology; Legal content.
\end{abstract}


Factores limitantes del diálogo entre los procesos de enseñanza-aprendizaje...

\section{Introducción}

La enseñanza de las ciencias jurídicas ha mantenido sus tradiciones pedagógicas en un enfoque centrado en el profesor. Él ha sido considerado como aquel que porta el conocimiento y lo transmite al estudiante, lo que se explica por la presencia de modelos psicopedagógicos tradicionales, basados en fundamentos positivistas y conductistas del aprendizaje. Este contexto educativo provoca inconvenientes a la enseñanza de las ciencias jurídicas, toda vez, que no se plantea situar al estudiante, en una posición de interacción activa, crítica y creativa con el objeto de conocimiento educativo jurídico, es decir, con las categorías, teorías y bibliografía que componen a las ciencias jurídicas como sistema teórico. Tampoco le otorga protagonismo para crear conocimiento o ser un pensador crítico y revisor epistémico.

Los estudiantes, por la recepción pasiva del conocimiento, y muchas veces influenciados por la clase magistral, meramente expositiva, como la clasificaría Elgueta Rosas y Palma González (2014), no logran comprender la profundidad de los debates, ni lo que el profesor implica con su relato. Puede en muchos casos, repetir los razonamientos, e incluso, llegar a aplicar la mayoría de los contenidos, pero el problema está, y es el interés de este trabajo demostrarlo, en la posición pasiva que se promueve, para que interactúen con ellos, y de hacerlo intersubjetivamente en el aula.

Esto provoca como consecuencia, lo que denominamos una discapacidad dialógica, es decir, una dificultad para dialogar con las tramas categoriales, teorías y significados jurídicos, lo que le limita para construir fundamentos y dar soluciones a casos planteados de la vida profesional o simulaciones didácticas; en última instancia, para enfrentar una realidad desconocida y tener las habilidades para dar respuesta creativa a tales necesidades. No se debe olvidar que ello es de la esencia misma de un profesional y en especial de las ciencias jurídicas, dado que un abogado debe mediar la realidad desde categorías jurídicas.

Sin embargo, se deben reconocer los avances y la tendencia positiva a innovar en la enseñanza de las ciencias jurídicas en general ${ }^{1}$ y también en el aula de especialidad, como en las ciencias penales (Ríos Corbacho, 2012 ; Holgado Saéz, 2013; Íñigo Corroza, E. y Sánchez-Ostiz Gutierrez, 2014; Scheechler Corona,2012; Abel Souto, M. 2012) en el aprendizaje del Derecho Constitucional (Becerra Valdivia y Salas Salazar, 2015), en el estudio y enseñanza del Derecho Civil (Prayones, 2006 y Colmo, 2011) y

\footnotetext{
${ }^{1}$ En los últimos años académicos del área jurídica se han encontrado en Eventos científicos nacionales I, II, III y IV Congreso de Pedagogía y Enseñanza del Derecho realizados en la Universidad de Chile; el Seminario Nuevos paradigmas e innovaciones en la Enseñanza del Derecho, realizado por la Universidad Católica del Norte, Chile, 2011, ciudad de Antofagasta y Coquimbo (2014, 2015 y 2016) e internacionales, así como el Segundo Congreso Internacional de Pedagogía Universitaria y Didáctica del Derecho UNAM México 2014, entre otros sobre el tema.
} 
en metodologías para la enseñanza del Derecho del Trabajo y de la Seguridad Social (Pérez Campos, 2012), por ilustrar someramente algunas experiencias. Con ello se ha favorecido la incorporación de enfoques constructivistas, modelos de enseñanza centrados en el estudiante y didácticas activas, de acuerdo a las sugerencias dadas desde el Proyecto Tuning América-Latina, que convocan al abandono de la tradicional formación destinada a la memorización de las normas jurídicas, por una más práctica y crítica, que se proyecte a la formación de competencias. Debates y propuestas que se mantienen en polémica, sobre todo, por la preocupación que existe por la cantidad de abogados que se titulan anualmente (Lazo González, 2011).

Sin embargo, con todo, no se ha profundizado en la relación que se da entre estudiante y el objeto de conocimiento jurídico; con este sistema abstracto de teorías que significan, y desde ahí entonces, las interrogantes que surgen: ¿la enseñanza de las ciencias jurídicas, promueve una interacción activa con el contenido enseñado, es decir, con el objeto de conocimiento educativo jurídico?, y particularmente, ¿logra el estudiante interactuar con este sistema abstracto de categorías?; ¿lo construye y/o deconstruye, desde alguna posición?, ¿dialoga con él y se lo apropia, o simplemente lo recibe y asimila?

En consecuencia con lo anterior, el objetivo planteado en este trabajo de aproximación epistémica a la problemática planteada, ha sido indagar y examinar los obstáculos y límites, que una enseñanza tradicional reproductora promueve, para no desarrollar una relación de interacción de los estudiantes de Derecho, con el objeto de conocimiento jurídico. En ese contexto, se propone demostrar las consideraciones de tipo epistemológico y políticos, que impide desarrollar esta ésta necesaria interacción (estudiante-objeto), que permita, se dé una apropiación y, al mismo tiempo, la toma de conciencia de la trascendencia ideológica que ello implica.

\section{Límites que obstaculizan la relación del estudiante con el objeto de conocimiento educativo jurídico en un contexto de aprendizaje activo}

Para realizar un análisis epistemológico y político del objeto de conocimiento educativo jurídico, así como su composición -entendiendo ello, la síntesis de comunidades científicas en constante desarrollo-, es necesario establecer algunos aspectos a considerar, donde se propicia y surge lo que hemos denominado, discapacidad dialógica entre estudiante y objeto de conocimiento educativo jurídico.

Como primera aproximación epistemológica, se hace conveniente precisar, que la praxis de la enseñanza-aprendizaje jurídica, se ha centrado principalmente, en el contenido, lo que denominaremos objeto de conocimiento educativo jurídico. Éste corpus, compuesto por teorías, tramas categoriales, instituciones y normas jurídicas, 
Factores limitantes del diálogo entre los procesos de enseñanza-aprendizaje...

sumado a todo aquello, que compone un sistema artificial con significados específicos. En la práctica, consideramos que no se ha puesto atención, a cómo se relaciona el estudiante de Derecho con este sistema, o cómo se enseña a hacerlo, y a él, a aprender a aprenderlo.

Al respecto, la literatura pedagógica reconoce algunos tipos de estrategias de aprendizajes, en relación a cómo se interactúa con el objeto de conocimiento jurídico. Destacan, entre los más estudiados, el llamado aprendizaje superficial, al cual se contrapone, el denominado aprendizaje profundo. El primero, propiciaría métodos de enseñanza, que reproducen particularidades de un contenido determinado, donde el estudiante acepta ideas, información y memorización de manera rutinaria (Salas Silva, 1998), mientras que el segundo, sería una estrategia de aprendizaje, generador de movilidad y flexibilidad, pues sobre un mismo tópico, se puede realizar distintas operaciones mentales (Beas Franco, Manterola, Pacheco y Santa Cruz Valenzuela, 1998), lo que no sólo implica logros en comprensión profunda (Valenzuela Carreño, 2008), sino además, el beneficio de conseguir una retención en el largo plazo, para permitir que puedan ser utilizadas en la solución de problemas en contextos diferentes (Fasce Henry, 2007).

Se observa entonces, que la praxis de la enseñanza jurídica centrada preponderantemente en el contenido, ha promovido y desarrollado un aprendizaje del tipo superficial, que tiene como resultante, a estudiantes poco preparados para comprender la profundidad de lo que debate, ni lo que está de fondo, como son los intereses ideológicos o valoraciones, sobre determinada problemática. Fasce Henry (2007), al respecto, establece que esta forma de aprendizaje, conlleva la memorización de la información como hechos aislados y sin conexión con las experiencias previas o con el contexto general, señalando además, que el objetivo central consiste en retener datos para aprobar la evaluación.

De lo anterior se desprende, que desde una estrategia de enseñanza superficial, el estudiante no aprende a interaccionar con el objeto de conocimiento educativo jurídico, más bien lo recepciona pasivamente. Ello, va en la línea de lo planteado por Freire (2012), quien cataloga como educación bancaria, a aquella praxis donde el docente no se comunica con los estudiantes, no abre espacio a dialogar con los contenidos a aprender, sino más bien deposita en ellos comunicados y donde la única acción que se le ofrece es recibir, guardar y depositar.

\subsection{Relación epistemológica sujeto-objeto de conocimiento en las diná- micas curriculares; lo que dice relación con el qué conocer (Gómez Fran- cisco, 2010)}

Otro de los límites, que a nuestro entender, obstaculizan la relación del estudiante con los contenidos del aula, está dado con la consideración epistemológica 
del objeto de estudio, del sujeto cognoscente y de cómo se da la relación en el aula jurídica. Al respecto, es necesario establecer, que epistemológicamente, la relación dialéctica sujeto-objeto, ha descrito una historia dinámica, que resurge en la modernidad, con la clásica posición cartesiana que establece la dualidad entre ambos, hasta la participación dialéctica que ha venido a desarrollarse desde las distintas revoluciones científicas. Así, desde la Física con Heisenberg (1976), intenta demostrar la dificultad del sujeto para desprenderse de su objeto, lo que termina por incorporarse en cierto sentido en él, lo mismo hizo Maturana Romesín (1997) desde la Biología, considerando también, el avance registrado desde las Ciencias Cognitivas, el Constructivismo y la Cibernética de Segundo Orden.

Desde paradigmas emergentes, como los individualizados anteriormente, se ha cuestionado al modelo positivista -principal protagonista en la investigación educativa en general y de la enseñanza jurídica en particular-, visto como el promotor de una pretendida objetividad y neutralidad investigativa, que tiene como resultante en el aula jurídica, el conocer y reproducir lo que dijo algún teórico validado por la tradición del área, lo que se intenta reproducir con el mayor celo.

El objeto de estudio, desde una visión mecanicista del mundo, ha sido planteado como una colección de objetos, donde cualquier interacción entre ellos, aparece como una relación secundaria. A este objeto, desde los paradigmas clásicos positivistas, se le otorgó la cualidad de ser medible y con el método de descomposición, se permite experimentar y manipularlo (Capra, 1992). Además, su existencia es atomizada y autosuficiente, en consecuencia, se conoce mejor cuando se le aísla. Así entonces, la noción de objeto de conocimiento, concebido desde la ciencia clásica, se caracteriza, al mismo tiempo, por estar fuera del sujeto cognoscente, puesto para ser descubierto (Pérez Soto, 1998).

En la praxis de la enseñanza jurídica, siguiendo este patrón, tenemos que el conocimiento jurídico es entregado por un docente que aparece como neutral, no percibiéndose además, que el contenido transmitido ha sido significado por él. En tal sentido, para este objeto de estudio jurídico, no existe conciencia que es obra de alguien, quien subjetivamente entramó normas, principios y autores, de una manera particular. Ante ello, es conveniente sintetizar, que el objeto-contenido que conocen los estudiantes, no es neutral, por el contrario, es una intervención del docente; es su obra, pues lo intervino, se plasmó en él, eligió qué contenido, qué autores, qué tema enfatizar, qué instruir, en síntesis es también una valoración avalada desde una trama de poder -según una noción de Foucault (1993) de éste-, de ahí la significancia política, que está a la base de la praxis de enseñanza jurídica clásica.

Como se aprecia, en la enseñanza tradicional del Derecho, por lo general se sigue asumiendo el objeto de estudio jurídico, desde una visión mecanicistapositivista, caracterizada por enfatizar la fuente formal, por dividir el corpus jurídico 
Factores limitantes del diálogo entre los procesos de enseñanza-aprendizaje...

en artículos atomizados, y desde ellos, pretender avanzar a dimensiones más complejas, de manera lineal y mecánica. En ese sentido, seguir admitiendo al objeto de conocimiento científico -jurídico-, desde una perspectiva clásica, implica limitar la interacción del estudiante con él, pues lo asume como algo encapsulado, que debe percibir como una cosa que debe memorizar y decir o repetir en una evaluación, de ahí que el sujeto cognoscente, en esta lógica, sea visualizado como un recipiente a ser Ilenado, siguiendo la analogía de Benfeld Escobar (2016, p. 161), o la de los recipientes vacíos señalados por Coloma Correa (2005, pp. 143-145).

De ahí, que las metodologías se centren en esquematizar contenidos, para que sea fácil recordar, memorizar palabras claves, donde los sentidos como la vista y oído, adquieren un protagonismo relevante. Así entonces, el objeto a conocer, se transforma en un reflejo a digerir, al que hay que acercársele y en lo posible asimilarlo. Esta experiencia es un hábito en la relación social educativa jurídica. Al respecto, Nino (2004, pp. 11-12) desataca que en el Derecho, el sólo preguntarse por lo que es, ya causa escozor. Esto se debe a que en nuestro ámbito, predomina fuertemente la concepción platónica de la relación lenguaje-realidad. De esta forma un concepto debe reflejar una realidad, siendo las palabras una suerte de espejo de aquella objetiva realidad en la que el hombre no puede interferir, sino solo captar lo esencial que, ineludiblemente, debe ser recogido por el concepto.

En este contexto, desde un ideal científico clásico, también el sujeto cognoscente pretende mantener una posición ajena, neutral y distante del objeto a conocer; no lo interviene, sino que lo observa, lo analiza y lo descubre. Desde esa perspectiva, el sujeto no añade nada nuevo a la realidad que indaga, pues sus sensaciones y percepciones se limitan a "reflejar" las propiedades de los objetos indagados (Sotolongo Codina, P. y Delgado Díaz, 2006, pp. 49-50). Esta lógica, llevada al caso del sujetocientífico jurista clásico, asume la legislación como su objeto de estudio; la ordena e interpreta según criterios establecidos, bajo la premisa de eliminar del proceso toda subjetividad, en la configuración del objeto de estudio. Así entonces, la tendencia en el aula es partir de lo dado, de la fuente formal; pues todo lo demás -fuente material, la opinión de la doctrina, etc.- se considera marginal, secundario o accesorio, con lo que se niega el dialogo texto-contexto, objeto de conocimiento dado-dándose (Witker Velásquez, 2016).

Por el contrario, desde nuevos paradigmas emergentes, el objeto social de conocimiento, se le concibe -epistemológicamente- como una construcción, que resulta del desarrollo de la sociedad, como también, del estado de la investigación (Mejía Navarrete, 2002), lo que lo hace dinámico, relativo y en directa relación con el sujeto cognoscente. En particular, desde paradigma científico de la complejidad y del Pensamiento Complejo, se ha comenzado a admitir que los objetos a conocer, están más interrelacionados de lo que se había considerado. Al respecto, Morin (1986 pp. 117-127) señala que una organización representa la disposición de relaciones entre 
componentes o individuos que produce una unidad compleja o sistema, y que ello, contiene cualidades desconocidas en el nivel de los componentes o individuos. Así entonces, la conexión/desconexión, vinculación/desvinculación, de artículos, principios o jurisprudencia, adquieren una cualidad según se organicen. Ante lo cual, no da igual partir desde un argumento que valore de tal o cual manera la relación. Relacionar y significar información y argumentos, los convierten en una trama valorada que sólo se comprende desde un aprendizaje profundo, porque permite una posición dinámica con el objeto de conocimiento jurídico. La forma de conocer de un estudiante de Derecho, no podría ser un percibir, sino un concebir -desde una práctica situada y desde un conocimiento previo-.

\subsection{La noción de ciencia jurídica como producto; no como actividad y proceso}

Un factor relevante, que actúa como límite obstaculizador, en la relación del estudiante con el objeto de estudio jurídico, es la asentada noción de observar la ciencia como producto creado por alguien aislado: un científico, un experto propietario de un saber. En ese sentido, no está valorizado que las ciencias jurídicas, representan la síntesis de una actividad científica de intersubjetividad, desarrollada por comunidades científicas, que además, establecen disputas internas y externas, desarroIlan intereses, acuerdos y contradicciones, como también, promueven un proceso de reinvención, como manifiesta Coloma Correa (2016).

En estas dinámicas, por cierto que se debaten y coinciden con ciertos consensos y/o paradigmas, lo que devela la dificultad de admitirlos a priori neutrales. De este activo proceso, se van habituando ciertas ordenaciones clasificatorias, familiarizando ciertos autores, repitiendo posiciones doctrinarias que se van consolidando como un corpus que en la mayoría de los casos, es preservado en manuales, naturalizando con ello, algunas posiciones que posteriormente, son enseñadas. Visto así, la ciencia jurídica aparece como producto destinado a ser traspasado, desde un científico-experto, hacia un aprendiz pasivo.

Esta visualización, omite la actividad y proceso implícito de la enseñanzaaprendizaje de la ciencia jurídica, donde convergen diversas dinámicas de las comunidades científica-jurídicas, lo cual no permite además, reconocer el poder que tienen, en la selección del objeto de conocimiento jurídico, antecedente, que de ser conocido y socializado entre los estudiantes, pudiese otorgar una mejor proyección al objeto de estudio jurídico, promoviéndose una apertura dialogante, con cabida para la incorporación de distintas perspectivas, cosmovisiones y paradigmas.

Lo cierto, es que en general, la concepción de la ciencia como producto, ha venido siendo resistida y discutida, desde los paradigmas emergentes. En concreto, desde la década del sesenta del siglo pasado, esta concepción, de manera acumulativa, ha entrado en discusión, principalmente, a partir de epistemólogos que han ve- 
Factores limitantes del diálogo entre los procesos de enseñanza-aprendizaje...

nido a demostrar la importancia de las comunidades científicas en la producción del conocimiento científico. Entre los clásicos ya en estas materias está Kuhn (1998), a través de la explicación de paradigmas y revoluciones científicas y Lakatos (1993) con la explicación de los Programas de Investigación. Ante esta apertura ya se han incorporado investigaciones que estudian sus dinámicas teóricas e ideológicas (Casas Guerrero, 1980), en lo cual, por cierto, que la ciencia jurídica está al debe.

\section{2. ¿Qué se está enseñando como objeto de conocimiento jurídico; cuáles son las representaciones que se tienen sobre éste, que limita un diálogo activo con él?}

Cuando el objeto educativo es la ciencia -en este caso ciencias jurídicas-, se hace necesario indagar cuáles son las representaciones comunes que tienen los docentes sobre la ciencia, y que causa desmotivación, poca participación y una desvinculación del estudiante con el objeto de estudio jurídico. Al respecto, Jorge Molina (2009) ha distinguido algunas de estas representaciones, partiendo por establecer que los docentes, tienen por representación a una ciencia que descubre realidad, lo que trae aparejado una noción de la verdad, que por cierto, viene a avalar todo lo que implica el desarrollo teórico, que le permite al investigador clasificar, ordenar y nombrar, por ejemplo, las normas jurídicas. En razón de lo anterior, surge la pretensión que las ideologías y creencias, no afectan el conocimiento, porque en principio, debiera ser igualmente visto por todos quienes realizan ciencia y siguen su método.

El mismo autor, plantea la representación que establece a la ciencia, como la que investiga y descubre leyes, dado que al estar la realidad entregada al descubrimiento científico, como lo está un organismo vivo para un biólogo, o un psicótico para un psicólogo, se comprende como un "algo" objetivo, generalizado como válido, y con cierto grado de independencia de debates contingentes, lo que para las ciencias jurídicas será el conjunto de normas jurídicas. En esa misma línea, aparece la ciencia como acumulativa y lineal, al ser realizada por individuos que van descubriendo la realidad, se va acumulando conocimiento como una suma, pero no en saltos ni en rupturas de paradigmas; no se reconoce la intervención de las comunidades científicas, ni recursividad, caos e incertidumbres en los objetos de estudio, ni en el proceso de construirlos.

En este sentido, en el ámbito jurídico, resulta paradigmático el caso de la Política Criminal y sus dificultades para emanciparse como un área especial de conocimiento de las Ciencias Penales. Pues bien, visto desde la plataforma que aquí se propone, resulta sugestiva una perspectiva rizomática (Deleuze, G. y Guattari, 2002, pp. 11-29) como base epistemológica desde donde sustentarse para observar y analizar la Política criminal. Así, no se trataría sólo de una panorámica multidisciplinaria, sino de un cambio de paradigma en la forma de entender el conocimiento, es decir la 
ciencia. De esta manera, ya no desde una visión arbórea, sino precisamente desde su antípoda, con una percepción no fundacional, puede entenderse mejor el funcionamiento de una Política criminal donde las fronteras de las ciencias que la conforman, antes que separarlas, las une. De este modo, el funcionamiento del sistema político criminal no dependería de un centro desde el cual se ramifiquen otros conocimientos subsidiarios, evitando así la superioridad de una disciplina en desmedro de las otras, y provocando que la elaboración del conocimiento surja simultáneamente desde todos los puntos, bajo la influencia recíproca de las distintas observaciones y conceptualizaciones. Desde una visión tradicional lo plantado desborda los márgenes de su epistemología. De dicha manera se entiende, que desde una plataforma convencional, antes que poder formularse una teoría apenas se deje constancia de un asombro (Cardozo Pozo, 2009).

Molina (2009), afirma también en este sentido, que 1) el conocimiento se transforma en producto, donde se construye una representación realista de él, 2) la experiencia se transforma en la base del conocimiento, el sujeto cognoscente queda subordinado al registro perceptual, dejando fuera la creatividad, reflexión, 3) la observación como base de la actitud científica, dejando fuera las ideologías, valores, en definitiva, la subjetividad.

Lo anterior, para la formación del abogado, implica un abandono de la práctica reflexiva, dificultando con ello, habilidades para crear casos o estrategias jurídicas. Esta pasividad que se reproduce como hábito educativo y que porta muchas veces el estudiante, no permite que el profesor se disponga a proporcionar herramientas, que le permitan organizar y discriminar información, interpretar y construirla - ¿para qué?-. Esto, en cualquier ámbito educativo, donde está comprometida una ciencia, es necesario que al menos, sea revisada, más aún, en las ciencias jurídicas, en tanto, adquieren una connotación especial, por su relación con el poder.

La relación que se da entonces, entre el estudiante y el saber jurídico es distante, y su representación es realista como señala el autor. Lo anterior, provoca que para el proceso de enseñanza-aprendizaje, adquiera relevancia la percepción sensorial por sobre la reflexión, para la adquisición de conocimiento. Con esta concepción epistemológica, que está a la base del saber jurídico, como corpus del docente, se eluden, como advierte Aznar Minguet (1992), las reflexiones sobre el qué es, el por qué o para qué del conocimiento, como parte de un pensamiento cuestionador. Por el contrario, se da una adequatio intellectus et rei, es decir, una convicción que la verdad corresponde a la realidad, y donde no existe intervención, ni significación del interprete, sino más bien un reflejo. Molina (2009), por su parte, ante la representación que transmiten los docentes y que profundiza la neutralidad, propone una enseñanza de la ciencia con narrativa, que la presente con los significados construidos socialmente, con sentido y contexto. 


\section{Existe una subvaloración de la riqueza relacional del conocimien- to}

Enseñar ciencia tiene su particularidad, pues representa un proceso sintetizado que se compone de tramas categoriales, de entramados teórico-empíricos, de paradigmas científicos, etc. Razón por la cual, se requiere de un proceso de aprendizaje no conductista, es decir, no tiene que ver con desarrollar respuestas a ciertos estímulos -lo que en la educación del Derecho sería sentarse en un examen y repetir palabras de corrido ante la pregunta de un docente -, sino, por el contrario, la praxis de la enseñanza-aprendizaje jurídica, se relaciona con el desarrollo de procesos, que implique una relación dialógica y mediada entre el sujeto y objeto de conocimiento, desde la cual, la verdad científica ya no sea considerada un producto dado, sino contextualizado. No se da un saber por correspondencia con el objeto investigado, sino un proceso de construcción práctica, lo que implica descartar la existencia per se de la verdad, como señala Gallego Badillo (1996, p. 264).

Pertinente a esta fundamentación, es el aporte de Sotolongo Codina y Delgado Díaz (2006, p. 56), quienes afirman la existencia de un proceso cognitivo que transcurre inmerso en una inter-subjetividad, dado que no existen "robinsones" cognitivos, pues cuando se investiga, se asume lo que dijo tal o cual colega, autor, teoría y/o posiciones de otros. Al mismo tiempo, se da una inter-objetividad, donde investigar un objeto, implica que nunca se puede aislar; por el contrario, ese objeto es en su articulación, es con múltiples otros objetos del mundo social -jurídico-. Es decir, una investigación científica -el qué enseñar y por ende, con qué dialogar- se da como un proceso de intersubjetividad e interobjetividad, pues "... la construcción del mundo, tiene lugar, no dentro de la mente del observador, sino en las formas de relación" (Molina, 2012, p. 380). Esta verdad construida por consenso intersubjetivo, como verdad contextual, valida no sólo el sentido pedagógico de reconocer la importancia de las ideas previas que traen los estudiantes, y que contribuye al proceso de enseñar la ciencia, como lo han demostrado investigaciones (Monroy Nasr, León Sánchez y Álvarez Díaz de León, 2012) y en particular el Derecho, como lo establecen Fairstein, Scavino, Frontini, Torre, Duhalde y Potenze (2014), sino también, convoca estudiantes interactuantes, y para aprender a serlo. Así, la educación como metáfora de la vida, tiene mucho que hacer en el logro de un saber integral incluyente en la búsqueda de la verdad (Pupo Pupo, 2014).

Entonces, no admitir la dinámica relacional, que porta el conocimiento y, por ende los objetos de conocimiento, con los que debe interactuar un estudiante de Derecho, implica un reduccionismo, que afecta cualquier proceso pedagógico, que tenga a la ciencia como objeto de conocimiento educativo. De ahí surgen interrogantes insoslayables a considerar: ¿se estará dimensionado, para la enseñanza del Derecho, la complejidad del objeto de conocimiento jurídico como algo dinámico y contextuado?; ¿los sujetos involucrados, tanto docentes como estudiantes, se en- 
cuentran habilitados para dialogar con la ciencia jurídica, como conocimiento en construcción?

En definitiva, se observan límites epistemológicos, pedagógicos e incluso políticos que limitan la interacción del estudiante de Derecho con el objeto de su conocimiento (Kennedy, 2012, p. 110). Por lo tanto, promover un acercamiento de las partes, requiere replantearse el ámbito unidireccional educativo, hacia una interacción participativa. A ello, se debe abocar esfuerzos pedagógicos e incentivar la toma de posición en las dimensiones científicas.

\section{Poca claridad sobre la composición del objeto de conocimiento educativo jurídico -el qué enseñar-}

Hemos señalado anteriormente, que el objeto de estudio de la ciencia jurídica, está constituido por tramas categoriales que requieren, para su comprensión, que los estudiantes desarrollen un pensamiento que les permita interactuar con ellas, pero que al mismo tiempo, logren desarrollar una comprensión profunda de sus significados e implicancias contextuadas. En ese sentido, Carretero Rodríguez (2000, pp. 97-98) advierte que el conocimiento científico posee algunas diferencias respecto del conocimiento cotidiano y que a la luz de la composición del objeto de conocimiento educativo jurídico, es sugerente conocer. A saber, el autor señala que acercarse al conocimiento científico, obliga tener un alto nivel de abstracción, pues los conceptos científicos no siempre tienen un referente concreto en la realidad cotidiana y por lo tanto, no se pueden percibir directamente. Esta observación del autor, permite comprender por ejemplo, la dificultad que se tiene para comprender la nulidad como sanción jurídica, la verdad formal, o que se debata si los animales son cosa mueble o sujetos de Derecho.

Carretero Rodríguez (2000, pp. 97-98) también señala que el conocimiento científico, posee una estructuración de los conceptos en forma de teoría, ya que éste utiliza nociones que sólo adquieren sentido en el entramado de teorías estructuradas; por ejemplo, el injusto en la teoría del delito, el acto jurídico, categorías como propiedad, familia, hijos, derechos subjetivos por ilustrar algunos. Además, señala el autor, que en el conocimiento científico, los contenidos son contrarios a la intuición cotidiana, pues en muchas ocasiones, la ciencia plantea teorías que se oponen a nuestras experiencias; en el caso de las ciencias jurídicas, podría resultar la absolución del homicidio de una persona que dio muerte a otra, sólo por vicios procesales ${ }^{2}$.

Tras lo revisado, es conveniente considerar, que el estudiante de Derecho debe tener las herramientas cognitivas y relacionales para navegar en este mar, a veces turbulento, de categorías, significantes y significados. Poseer además, una movilidad

\footnotetext{
${ }^{2}$ N. R. Los ejemplos jurídicos son de los autores.
} 
entre un pensamiento abstracto/ concreto, común/científico. De ahí entonces, que para que exista claridad sobre la composición del objeto de conocimiento educativo jurídico, vale decir, que el docente entienda el qué enseñar, y el estudiante el qué aprender, se debe desarrollar un contexto educativo, con capacidad dialógica, que permita comprender los fundamentos epistemológicos y políticos de las ciencias jurídicas.

\section{Conclusiones}

Como hemos señalado, las ciencias jurídicas no corresponden a una verdad encapsulada, sino una trama compleja de relaciones categoriales, significados y valoraciones, lo que produce que sus interacciones no sólo representen conexiones lógicas y fundadas, sino además, connotaciones ideologías y/o cosmovisivas. Si ello, es advertido por los actores educativos en el contexto de aula, como una disposición especial para fortalecer una comprensión crítica, abre espacios para desarrollar una relación intersubjetiva con el contenido jurídico, lo que traería implícito, una metacognición, es decir, una autorregulación del pensamiento, o un conocimiento sobre los propios procesos y productos cognitivos, o cualquier otro asunto relacionado con ellos, como son los factores motivacionales, lo que se reconoce como aprender a aprender, saber qué se debe corregir, cuáles son los límites y cómo mejorarlos (Campanario Larguero, 2000).

Así entonces, el desafío que se propone para romper los límites obstaculizadores, entre el estudiante de Derecho y el contenido jurídico, entendido como su objeto de conocimiento educativo, implica comenzar a transformar el ámbito de las relaciones sociales educativas en el aula de las ciencias jurídicas, de tal manera, que el aprendizaje, sea un proceso de diálogo consciente de intersubjetividades, contando no sólo docentes-estudiantes-estudiantes-docentes, sino también con los autores que participan por escrito y con teorías, que representan posiciones o síntesis de controversias al interior de las comunidades científicas, con manifestación de posiciones e intereses. $Y$ donde todo objeto es dinámico organizado y organizable de conocimiento educativo jurídico como interobjetividad; no son piezas, átomos, artículos aislables, sino entramados complejos.

Para que el futuro profesional logre desarrollar conocimientos que le permitan tener movilidad y pueda administrarlos según el contexto, su requerimiento y principios, deben dilucidar en su proceso de formación formal, la mayor parte de las interrogantes que se generan desde el saber jurídico; qué se conoce, cómo y para qué (Gómez Francisco, 2014). Los sujetos cognoscentes, no deberían seguir relacionándose con el contenido de su aprendizaje, como si fueran objetos acabados y perceptibles por los sentidos. Por el contrario, deben aprender a relacionarse con él, teniendo claro que es un objeto relacional, tanto en su origen intersubjetivo y en su 
composición entramada de teorías, categorías e intereses. Para ello, se debe promover un contexto intersubjetivo de aprendizaje activo, el desarrollo de un pensamiento abstracto capaz de dialogar con tramas categoriales y con una ciencia no neutral. Con esto, se promueve un aula democrática, donde los sujetos dialoguen con un objeto de estudio que no se representa como una verdad reflejada, sino por el contrario, un construible dinámico. Todo sobre la base de un proceso psicopedagógico orientado a crear escenarios posibles, para que la relación docentes-estudiantesestudiantes-docentes, se transforme en un diálogo práctico, una intersubjetividad contextuada.

De ahí, que el objeto de estudio no debe ser entendido intuitivamente en correspondencia con la realidad, sino, como una creación científica, lo que exige articulación de argumento, fundamentos, creatividad y estrategia, lo que implica dejar de lado metodologías lineales y mecanicistas, que propician aulas jurídicas atomizadas, donde el conocimiento es entendido como un algo cosificado; un producto a entregar y/o recibir. Por el contrario, se debe dar paso a estrategias de aprendizaje, que permitan el movimiento recursivo del pensamiento y conectadoras de categorías y significaciones; propiciadoras de procesos de construcción e indagación científica, donde converjan el texto y contexto, con un objeto de estudio dado y dándose.

Lo anterior, implica eliminar la neutralidad y objetividad del objeto de estudio, ya que en el dinamismo dialógico de la praxis jurídica, existe presencia de contingencia, paradigmas emergentes, comunidades científicas y valoraciones. Entender ello, es preparar al futuro abogado, para interactuar con la creatividad, las emergencias e incertidumbres; para lidiar con la verdad-poder, que implica comenzar a deconstruir la dualidad sujeto-objeto, los contenidos jurídicos estancos, objetuales y neutrales, y por cierto, las concepciones epistemológicas, que no admitan objetos relacionales complejos.

\section{Agradecimientos}

Este artículo contiene resultados de un proyecto de investigación interno de la Universidad de Atacama de Chile (DIUDA), denominada "Cambios epistemológicos y acercamientos disciplinarios en un proceso educativo". Y de estudios realizadas por el Programa para la Enseñanza Integral del Derecho PEID-UDA.

\section{Referencias Bibliográficas}

Abel Souto, M. (2012). Desde la literatura, ópera, cine y televisión hasta las ciencias jurídicas y el Derecho Penal mediante la nueva técnica pedagógica del aprendizaje basado en problemas. Revista jurídica de investigación e innovación educativa, (5), 87-104. Recuperado de https://bit.ly/2qVCw6u 
Factores limitantes del diálogo entre los procesos de enseñanza-aprendizaje...

Aznar Minguet, P. (Coord.). (1992). Constructivismo y educación. Valencia: Tirant Lo Blanch.

Beas Franco, J., Manterola, Pacheco M., y Santa Cruz Valenzuela, J. (1998). Habilidades cognitivas y objetivos transversales: un tema para pensar y actuar. Pensamiento Educativo. 22(1), 175-192. Recuperado de https://bit.ly/2rP9kyr

Becerra Valdivia, K. y Salas Salazar, C. (2015). Sistema metodológico integral del aprendizaje del derecho constitucional a partir de la vivencia: una red de estrategias didácticas. Revista pedagogía universitaria y didáctica del derecho, 2(1), 68-82. https://doi.org/10.5354/0719-5885.2015.36682.

Benfeld Escobar, J. (2016). La discusión sobre la enseñanza del Derecho en Chile dentro del nuevo paradigma universitario: una tarea pendiente. Revista de Derecho (Coquimbo. En línea), 23(1), 143-171. https://doi.org/10.4067/S071897532016000100007.

Campanario Larguero, J. (2000). El desarrollo de la metacognición en el aprendizaje de las ciencias: estrategias para el profesor y actividades orientadas al alumno. Revista enseñanza de las ciencias, 3(18), 369-380. Recuperado de https://bit.ly/2qg7YMD

Capra, F. (1992). El punto crucial. Buenos Aires: Troquel.

Cardozo Pozo, R. (2009). Bases de política criminal y protección penal de la seguridad vial (especialmente sobre el artículo 379 del código penal) (Tesis Doctoral). Universidad de Salamanca. Recuperado de https://bit.ly/33OzfUU

Carretero Rodríguez, M. (2009). Constructivismo y educación. Buenos Aires: Paidós.

Casas Guerrero, R. (1980). La idea de comunidad científica: su significado teórico y su contenido ideológico. Revista mexicana de sociología, 42(3), 1217-1230. https://doi.org/10.2307/3539999.

Colmo, A. (2011). Sobre didáctica del derecho civil. Academia (Buenos Aires), 9(17), 115-145. Recuperado de https://bit.ly/2q79IrH

Coloma Correa, R. (2005). El ocaso del profesor Binns. Un ensayo acerca de la enseñanza del derecho en Chile. lus et praxis, 11(1), 133-172. https://doi.org/10.4067/S0718-00122005000100006.

Coloma Correa, R. (2016). Las disciplinas jurídicas y su reinvención. lus et praxis, 22(2), 253-298. https://doi.org/10.4067/S0718-00122016000200009.

Deleuze, G. y Guattari, F. (2002). Mil mesetas: capitalismo y esquizofrenia. (J. Pérez Vázquez y U. Larraceta, Trads.) (5a ed.). Valencia: Pre-Textos. 
Kennedy, D. (2012). La enseñanza del derecho como forma de acción política. (T. Arijón y G. Moro, Trads.). Buenos Aires: Siglo XXI.

Elgueta Rosas, M. y Palma González, E. (2014). Una propuesta de clasificación de la clase magistral impartida en la facultad de derecho. Revista chilena de derecho, 41(3), 907-924. https://doi.org/10.4067/S0718-34372014000300006.

Fairstein, G., Scavino, C., Frontini, P., Torre, V., Duhalde, M. y Potenze, M. (2014). La construcción del conocimiento en ciencias jurídicas y su valor para la enseñanza. Academia (Buenos Aires), 12(23), 181-190. Recuperado de https://bit.ly/33Rz3Ey

Fasce Henry, E. (2007). Aprendizaje profundo y superficial. Tendencias y perspectivas. Revista de educación en ciencia salud, 4(1), 7-8. Recuperado de https://bit.ly/2qUm32o

Freire, P. (2012). Pedagogía del oprimido. (J. Mellado, Trad.) (2a ed.). Buenos Aires: Siglo XXI.

Foucault, M. (1993). Microfísica del poder (J. Varela y F. Alvarez-Uría, Trads.) (2a ed.). Madrid: La Piqueta. Recuperado de https://bit.ly/373HkqZ

Gallego Badillo, R. (1996). Discurso sobre el constructivismo. Bogotá: Magisterio.

Gómez Francisco, T. (2014). Paradigmas emergentes y crisis en la educación del derecho: algo más que didácticas. Revista pedagogía universitaria y didáctica del derecho, 1(1), 58-71. Recuperado de https://bit.ly/2NPWWGJ

Gómez Francisco, T. (2010). La dualidad sujeto-objeto y sus repercusiones en el derecho. Frónesis, 17(1), 69-84. Recuperado de https://bit.ly/2QkMg4C

Heisenberg, W. (1976). La imagen de la naturaleza en la física actual (A. Ferraté, Trad.). Barcelona: Ariel.

Holgado Sáez, C. (2013). Derecho y cine del genocidio: 7 títulos contemporáneos (2001-2011) para la docencia presencial del derecho penal e internacional público. Revista jurídica de investigación e innovación educativa, (8), 99-116. Recuperado de https://bit.ly/379wMqo

Íñigo Corroza, E. y Sánchez-Ostiz Gutierrez, P. (2014). Sobre el uso del método inductivo en la enseñanza del derecho penal, Revista jurídica de investigación e innovación educativa, (9), 9-22. Recuperado de https://bit.ly/2Ks9AtH

Kuhn, T. (1998). ¿Qué son las Revoluciones científicas? (J. Romo Feito, Trad.). Barcelona: Altaya.

Lazo González, P. (2011). Formación jurídica, competencias y métodos de enseñanza: premisas. Ius et praxis, 17(1), 249-262. https://doi.org/10.4067/S071800122011000100011. 
Factores limitantes del diálogo entre los procesos de enseñanza-aprendizaje...

Lakatos, I. (1993). La metodología de los programas de investigación científica (J. Zapatero, Trad.). Madrid: Alianza.

Maturana Romesín, H. (1997). La objetividad. Un argumento para obligar. Santiago: Dolmen.

Mejía Navarrete, J. (2002). Perspectiva de la investigación social de segundo orden. Cinta de Moebio. (14), 200-225. Recuperado de https://bit.ly/2QmZ9v9

Molina J. (2009). La ciencia como Narrativa y su enseñanza. En Z. Monroy Nasr y R. León Sánchez (Eds.), Epistemología, psicología y enseñanza de la ciencia (pp. 225-234), México, DF: UNAM.

Molina, J. (2012). Explicación de cómo se adquiere el conocimiento y sus implicaciones para la enseñanza de la ciencia. En Z. Monroy Nasr, R. León Sánchez y G. Álvarez Díaz de León (Eds.), Enseñanza de la ciencia (pp. 371-380). México DF: UNAM.

Monroy Nasr, Z., León Sánchez, R. y Álvarez Díaz de León, G. (Eds.). (2012). Enseñanza de la ciencia. México, DF: UNAM.

Morin, E. (1986). El método 1: La naturaleza de la naturaleza. (A. Sánchez y D. Sánchez García, Trads.) (2a ed.). Madrid: Cátedra.

Nino, C. (2003). Introducción al análisis del derecho. Buenos Aires: Astrea.

Pérez Campos, A. (2012). Metodología para la enseñanza del derecho del trabajo y de la seguridad social en el espacio europeo de educación superior. Anuario jurídico y económico escurialense, (45), 155-176. Recuperado de https://bit.ly/37d5jnJ

Pérez Soto, C. (1998). Sobre un concepto histórico de ciencia. Santiago: LOM.

Prayones, E. (2006). Las reformas en la Facultad de derecho método de estudio y enseñanza del derecho civil. Academia, 4(7), 293-322. Recuperado de https://bit.ly/37fcVG3

Pupo Pupo, R. (2014). La verdad como eterno problema filosófico. En su Filosofía, educación cultura y pluralidad discursiva ensayística (Hacia una visión cultural y compleja del saber). Tepic, Nayarit: ISIC. Recuperado de https://bit.ly/2QqwLYV

Ríos Corbacho, J. (2012). Sobre la metodología y herramientas en la enseñanza del moderno derecho penal. Revista jurídica de investigación e innovación educativa, (6), 55-80. Recuperado de https://bit.ly/359PSL3

Salas Silva, R. (1998). Enfoques de aprendizaje entre estudiantes universitarios. Estudios pedagógicos (Valdivia), (24), 59-78. https://doi.org/10.4067/S071807051998000100005. 
Scheechler Corona, C. (2012). Uso de casos como recurso didáctico y formación por competencias en derecho penal. En Actas del primer congreso nacional de pedagogía universitaria y didáctica del derecho (pp. 271-290). Santiago: Universidad de Chile.

Sotolongo Codina, P. y Delgado Díaz, C. (2006). La revolución contemporánea del saber y la complejidad social: hacia unas ciencias sociales de nuevo tipo. Buenos Aires: CLACSO. Recuperado de https://bit.ly/2KsAVfe

Valenzuela Carreño, J. (2008). Habilidades de pensamiento y aprendizaje profundo. Revista iberoamericana de educación, 46(7), 1-9. Recuperado de http://bit.ly/2CMlxGm

Witker Velásquez, J. (2016). Los derechos humanos: nuevo escenario de la investigación jurídica. En E. Cáceres Nieto (Coord.). Pasos hacia una revolución en la enseñanza del derecho en el sistema romano-germánico (Vol. 2, pp. 423-438). México, DF: UNAM. Recuperado de https://bit.ly/2roAzp4

\section{Para citar este artículo bajo Norma APA 6a ed. \\ Gómez Francisco, T, Cardozo Pozo, R y Rubio González, J. (2019). Factores limitantes del diálogo entre los procesos de enseñanza-aprendizaje y el contenido jurídico, en la educación del derecho. Revista de Derecho (Coquimbo. En línea), 26, e3857. https://doi.org/10.22199/issn.0718-9753-2019-0013}

Copyright del articulo: @2019 Taelí Gómez, Rodrigo Cardozo y Juan Rubio

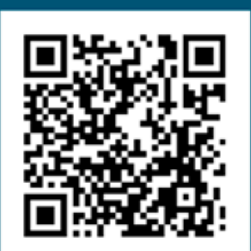

DOI

Este es un artículo de acceso abierto, bajo licencia Creative Commons BY 4.0. 